Pacific Journal of Mathematics

AN UPPER BOUND FOR THE PERIOD OF THE SIMPLE 


\title{
AN UPPER BOUND FOR THE PERIOD OF THE SIMPLE CONTINUED FRACTION FOR $\sqrt{D}$
}

\author{
R. G. Stanton, C. Sudler, JR. and H. C. Williams
}

Let $p(D)$ denote the length of the period of the simple continued-fraction expansion of $\sqrt{D}$, where $D$ is a positive non-square integer. In this paper, it is shown that

$$
p(D)<0.72 D^{1 / 2} \log D
$$

for all squarefree $D>7$, and an estimate for $p(D)$ is given when $D$ is not squarefree.

1. Introduction. The problem of finding a good upper bound for the length $p(D)$ of the period of the simple continued fraction for $\sqrt{D}$, where $D$ is a positive nonsquare integer, has received relatively little attention. Recently, Hickerson [6] and Hirst [7] have given estimates for $p(D)$; Hickerson's estimate implies that

(1.1) $\log p(D)<\log D\left(1 / 2+\log 2(\log \log D)^{-1}+o(\log \log D)^{-1}\right)$ ，

where $D$ is nonsquare, and Hirst's implies that

$$
p(D)<2 D^{1 / 2} \log D+0\left(D^{1 / 2}\right),
$$

"where $D$ is squarefree. Both authors give more precise error terms, but these are not relevant here. For general nonsquare $D>0$, Hirst shows that

$$
p(D)=O\left(D^{1 / 2} s \log D\right)
$$

uniformly in $s$, where $s^{2}$ is the largest square factor of $D$. For sufficiently large squarefree $D,(1.2)$ is clearly better than (1.1). On the other hand, (1.3) is better than (1.1) only when $s$, regarded as a function of $D$, is sufficiently small. Pen and Skubenko [14] have given an upper bound for $p(D)$ which we will discuss later; it depends on the size of the least positive solution of $x^{2}-D y^{2}=1$.

The authors [17] have used combinatorial methods to show that

$$
p(D)<0.82 D^{1 / 2} \log D
$$

for all squarefree $D>7$. In this paper, we use a different approach which refines this result to

$$
p(D)<0.72 D^{1 / 2} \log D
$$

for all squarefree $D>7$. It is also shown that

$$
p(D)<3.76 D^{1 / 2} \log \left(D / s^{2}\right)
$$


for all nonsquare $D>0$, where $s$ is defined in (1.3). The data in [1] suggest that $p(D)=o(D \log D)^{1 / 2}$.

It is clear that (1.5) is better than (1.1) for all large $D$. Moreover, (1.5) is an improvement on (1.3) in that it decreases, rather than increases, with $s$. When $D$ is squarefree, we also obtain a more precise theorem which implies that

$$
p(D)<A D^{1 / 2} \log D \cdot 2^{-\nu}
$$

for $D>1$, where $\nu$ is the number of prime factors of $D$ and $A$ is a computable constant. We conclude the paper by discussing the question of finding functions $g$ such that $p(D)>C g(D)$ for an infinity of $D$, where $g(D) \rightarrow \infty$ with $D$ and where $C$ is a positive constant.

We use the elementary theory of continued fractions and the theory of the units and class number of a real quadratic field as found, for example, in [2] or [11]. All small Roman letters denote positive integers unless otherwise stated; the phrase "continued fraction" always means "simple continued fraction".

2. A bound for $p(D)$ in terms of $L(1, \chi)$. We first prove a preliminary estimate. Suppose that $D$ is a squarefree integer $>1$. Then

$$
p(D)<\mu \log \varepsilon_{0} / \log \alpha
$$

where

$$
\begin{aligned}
\alpha & =(1+\sqrt{5}) / 2, \\
\varepsilon_{0} & =\left(u_{0}+v_{0} \sqrt{D}\right) / 2
\end{aligned}
$$

is the fundamental unit of $Q(\sqrt{D})$, and

$$
\mu=3 \text { if } 2 \nmid u_{0}, \quad \mu=1 \text { if } 2 \mid u_{0} .
$$

We note that, since $u_{0}^{2}-D v_{0}^{2}= \pm 4,2 \nmid u_{0}$ implies $D \equiv 5(\bmod 8)$; it follows from (2.4) that

$$
\mu=1 \text { if } D \neq \equiv 5(\bmod 8), \quad \mu \mid 3 \text { if } D \equiv 5(\bmod 8) .
$$

Now let $\left[q_{0}, \overline{q_{1}, \cdots, q_{p}}\right]$ be the continued-fraction expansion of $\sqrt{D}$, where $p=p(D)$; we have $q_{0}=[\sqrt{\bar{D}}]$. Further, if we formally define

$$
A_{-2}=0, \quad B_{-2}=1, \quad A_{-1}=1, \quad B_{-1}=0,
$$

and use the recursions

$$
A_{n}=q_{n} A_{n-1}+A_{n-2}, \quad B_{n}=q_{n} B_{n-1}+B_{n-2},
$$


for $n \geqq 0$, then $A_{n} / B_{n}$ is the $n^{\text {th }}$ convergent of the continued fraction for $\sqrt{D}$.

The relations $f_{0}=0, f_{1}=1, f_{m}=f_{m-1}+f_{m-2}$ for $m \geqq 2$, define the $m$ th Fibonacci number $f_{m}$. Hence we immediately obtain, by induction, the inequalities

$$
A_{n} \geqq f_{n+2}, \quad B_{n} \geqq f_{n+1},
$$

for $n \geqq-1$. Since $\alpha^{2}=(1+\sqrt{5})^{2} / 4=\alpha+1$, we find that $f_{n+2} \geqq \alpha^{n}$ for $n \geqq-1$; from (2.7), it follows that

$$
\eta=A_{p-1}+B_{p-1} \sqrt{D}>A_{p-1}+B_{p-1}>\alpha^{p},
$$

where $p=p(D)$.

A similar induction yields the better estimate

$$
A_{n} \geqq q_{0} f_{n+1}+f_{n} \quad(n \geqq 0) ;
$$

using the standard formula for $f_{n}$ in terms of $\alpha$, this produces

$$
\eta>\left(q_{0} / \sqrt{5}\right) \alpha^{p},
$$

as used in [17]. We later show that this sharper inequality (2.9) only improves Theorem 1 by an amount that is negligible when $D$ is large.

Now the least positive solution $\left(x_{1}, y_{1}\right)$ of $x^{2}-D y^{2}= \pm 1$ is $\left(A_{p-1}, B_{p-1}\right)$ : here we take the minus sign if $x^{2}-D y^{2}=-1$ is solvable; otherwise, we take the plus sign. Then the number $\eta$ in (2.8) is a unit in $Q(\sqrt{D})$; indeed,

$$
\eta=\varepsilon_{0}^{\prime \prime},
$$

where $\varepsilon_{0}$ is the fundamental unit of $Q(\sqrt{D})$ and $\mu$ is either 1 or 3 . Then (2.8) and (2.10) give

$$
p(D) \log \alpha<\mu \log \varepsilon_{0},
$$

as stated in (2.1).

We now apply a standard class-number formula to get the desired inequality for $p(D)$ in terms of $L(1, \chi)$. For squarefree $D>1$, the discriminant $\Delta$ of $Q(\sqrt{D})$ is given by

$$
\Delta=4 D \text { if } D \neq \equiv 1(\bmod 4), \Delta=D \text { if } D \equiv 1(\bmod 4) .
$$

It is known (see, for example, [2]) that

$$
\log \varepsilon_{0}=\sqrt{\Delta} L(1, \chi) / 2 h,
$$

where $h$ is the class number of $Q(\sqrt{D})$, 


$$
L(1, \chi)=\sum_{n \geq 1}(\Delta \mid n) n^{-1},
$$

and $(\Delta \mid n)$ is the Kronecker symbol (for a concise discussion of this symbol, see [4]). From (2.1) and (2.12), we obtain the

LEMma. Let $D$ be positive and squarefree; then

$$
p(D)<\mu \sqrt{\Delta} L(1, \chi) /(2 h \log c),
$$

where $\mu$ and $\alpha$ are given by (2.4) and (2.2) respectively.

We should here make some remarks about (2.14). It is easily proved by partial summation that $L(1, \chi)<A \log D$; so, by (2.14), we have $p(D)<B D^{1 / 2} \log D$ (here $A$ and $B$ are constants). In the next section, we will use an inequality due to Hua [8] to obtain an estimate for $L(1, \chi)$ of the above form with a better constant (for large $D$ ) than that given by the partial summation method alone.

The Riemann hypothesis for $L(s, \chi)$ implies that $L(1, \chi)=$ $0(\log \log D)$ [12, p. 367]; this result would give the estimate

$$
p(D)=0\left(D^{1 / 2} \log \log D\right) .
$$

On the other hand, it is known that $L(1, \chi)>C \log \log D$ for an infinity of squarefree $D$, where $C$ is a positive constant (see, for example, [9]). However, we do not know whether there is a positive constant $E$ such that $p(D)>E D^{1 / 2} \log \log D$ for an infinity of $D$; more generally, we do not know if (2.14) is sharp since we can not prove if there is a constant $F>0$ such that

$$
p(D)>F D^{1 / 2} L(1, \chi) / h(D)
$$

for an infinite sequence of squarefree $D$. (We shall return to the question of lower bounds for $p(D)$ in $\S 6$.)

It is easily seen that (2.15) can not hold for all nonsquare $D$. Since the right members of (2.1) and (2.14) are equal, (2.15) is equivalent to $p(D)>G \mu \log \varepsilon_{0}=G \log \eta$; so (2.9) implies that $p(D)>$ $H \log D$. Here $G$ and $H$ are positive constants. But $p(D)=1$ when $D=a^{2}+1$.

If we were to use (2.9) instead of (2.8), (2.14) would be replaced by

$$
p(D)<\frac{\mu \sqrt{\Delta} L(1, \chi)}{2 h \log \alpha}-\frac{\log \left(q_{0} / \sqrt{5)}\right.}{\log \alpha} ;
$$

we later show that (2.16) yields no significant improvement in (4.1) for large $D$.

We conclude this section by noting the estimate, due to Pen 
and Skubenko [14],

$$
p(D)<\log (T+U \sqrt{D}) / \log \alpha,
$$

where $(T, U)$ is the least positive solution of $x^{2}-D y^{2}=1$ and $D$ is squarefree. Now, $T+U \sqrt{D}=\eta$ or $\eta^{2}$ according as $x^{2}-D y^{2}=-1$ is not or is solvable (see, for example, [11]). Hence, by (2.10), $T+U V \bar{D}=\varepsilon_{0}^{\mu}$ or $\varepsilon_{0}^{2, t}$ according as the first or second alternative holds. So (2.17) is equivalent to

$$
p(D)<\mu \log \varepsilon_{0} / \log \alpha \quad \text { or } \quad p(D)<2 \mu \log \varepsilon_{0} / \log \alpha,
$$

respectively. Thus (2.1) is always at least as good at (2.17), and is sometimes better than it by a factor of 2 . Furthermore, our method is considerably simpler and more straightforward than that of [14].

Pen and Skubenko also give an inequality corresponding to (2.14) but they do not obtain any explicit numerical upper bound for $p(D)$.

3. Bounds for $L(1, \chi)$. Let $L(1, \chi)$ be given by (2.13), where $\Delta$ is now any nonsquare positive integer $\equiv 0$ or $1(\bmod 4)$. Hua has shown [8] that

$$
L(1, \chi)<1+\sum_{n=1}^{j} \frac{2|S(n)|}{n(n+1)(n+2)}
$$

where

$$
S(n)=\sum_{a=1}^{n} \sum_{m=1}^{a}(\Delta \mid m)
$$

and

$$
j=[\sqrt{\Delta}] .
$$

We note that $j \geqq 2$, since $\Delta \geqq 5$.

We first estimate $S(n)$ and consider three cases.

(i) $\Delta \equiv 0 \quad(\bmod 4)$. Then $(\Delta \mid 2 r)=0$ so that $|(\Delta \mid m)| \leqq$ $\left(1-(-1)^{m}\right) / 2$. It follows at once from (3.2) that

$$
|S(n)|<(n+1)^{2} / 4 \text {. }
$$

(ii) $\Delta \equiv 1(\bmod 8)$. Then we have trivially

$$
|S(n)| \leqq n(n+1) / 2 \text {. }
$$

(iii) $\Delta \equiv 5(\bmod 8)$. We estimate $L(1, \chi)$ for such $\Delta$ without using a bound for $S(n)$.

The sequence $D_{n}=1+1 / 2+\cdots+1 / n-\log n$ is easily proved to be monotone decreasing to Euler's constant $\gamma$; see, for example, 
[16], where it is proved that

$$
\frac{1}{2 n(n+1)}>D_{n}-D_{n+1}>\frac{1}{(n+1)(2 n+1)} .
$$

Then

$$
-1+\sum_{n=1}^{m-1}\left(D_{n}-D_{n+1}\right)=-1+\sum_{n=1}^{\infty}\left(D_{n}-D_{n+1}\right)-\sum_{n=k}^{\infty}\left(D_{n}-D_{n+1}\right) .
$$

Since $D_{n}-D_{n+1}=-1 /(n+1)+\log (n+1)-\log n$, we at once obtain

$$
\log k-\sum_{k=1}^{k} \frac{1}{n}=\lim _{k \rightarrow \infty}\left(\log k-\sum_{n=1}^{k} \frac{1}{n}\right)-\sum_{n=k}^{\infty}\left(D_{n}-D_{n+1}\right) \text {. }
$$

Thus

$$
\begin{aligned}
\sum_{n=1}^{k} \frac{1}{n} & =\log k+\gamma+\sum_{n=k}^{\infty}\left(D_{n}-D_{n+1}\right) \\
& <\log k+\gamma+\sum_{n=k}^{\infty} \frac{1}{2 k(k+1)},
\end{aligned}
$$

that is,

$$
\sum_{n=1}^{k} \frac{1}{n}<\log k+\gamma+\frac{1}{2 k} .
$$

We now apply (3.6) and our estimates for $S(n)$ to (3.1). Write $L$ for $L(1, \chi)$, and consider three cases.

Case 1. $\Delta=4 D \equiv 0(\bmod 4)$.

Substitute (3.4) into (3.1) to give

$$
\begin{aligned}
L & <\frac{1}{2} \sum_{n=1}^{j} \frac{1}{n}+1-\frac{1}{4}\left(1+\frac{1}{2}-\frac{1}{j+1}-\frac{1}{j+2}\right) \\
& <\frac{1}{2} \sum_{n=1}^{j} \frac{1}{n}+\frac{5}{8}+\frac{1}{2 j} .
\end{aligned}
$$

Apply (3.6)

$$
L<\frac{1}{4} \log D+\frac{1}{2} \log 2+\frac{1}{2} \gamma+\frac{5}{8}+\frac{3}{4 j} .
$$

Thus

$$
L<\frac{1}{4} \log D+1.28 \text { for } \quad \Delta=4 D, D>1500 \text {. }
$$

(Note that, in this section, $\Delta$ is any nonsquare positive integer $\equiv 0$ or $1, \bmod 4$, and so need not satisfy (2.11).) 
Case 2. $\Delta=D \equiv 1(\bmod 8)$.

By (3.1) and (3.5), we have

$$
L<1+\sum_{n=1}^{j} \frac{1}{n+2}<\sum_{n=1}^{j} \frac{1}{n}-\frac{1}{2}+\frac{2}{j} ;
$$

so we obtain, as before,

$$
L<\frac{1}{2} \log D+\gamma-\frac{1}{2}+\frac{5}{2 j} .
$$

Thus

$$
L<\frac{1}{2} \log D+0.09
$$

for $\Delta=D \equiv 1(\bmod 8)$ and $D>64,000$.

Case 3. $\Delta=D \equiv 5(\bmod 8)$. Here $(\Delta \mid 2)=-1$; hence

$$
L=\sum_{i=1}^{\infty}(D \mid 2 i-1)(2 i-1)^{-1} \sum_{k=0}^{\infty}(-2)^{-k},
$$

since the first series is convergent, and the second is absolutely convergent. Thus

$$
L=\frac{2}{3} \sum_{n=1}^{\infty}(d \mid n) n^{-1} \text {. }
$$

where $d=4 D$. Now use Case 1 and apply (3.7) to (3.10); this gives

$$
L<\frac{2}{3}\left(\frac{1}{4} \log D+1.28\right)<\frac{1}{6} \log D+0.86
$$

for $\Delta=D \equiv 5(\bmod 8), D>1500$.

4. Upper bounds for $p(D)$ when $D$ is squarefree. We use the preceding estimates for $L(1, \chi)$ to prove

THEOREM 1. Suppose that $D$ is squarefree and $>1$, and let $\mu$ and $\alpha$ be given by (2.4) and (2.2), respectively. Let $r$ be the number of distinct prime factors of $\Delta$, and set

$$
\begin{aligned}
& t=r-1 \text { if } D \text { is a sum of two squares, } \\
& t=r-2, \text { otherwise. }
\end{aligned}
$$

Thus $t \geqq 0$, and

$$
p(D)<\mu D^{1 / 2}(A \log D+B)\left(2^{t} \log \alpha\right)^{-1} \text { for } \quad D>64000,
$$

where the constants $\mathrm{A}$ and $\mathrm{B}$ are given by the following table. 


\begin{tabular}{ll|ll} 
& \multicolumn{1}{c}{$\mathrm{A}$} & \multicolumn{1}{c}{$\mathrm{B}$} \\
\hline$D \equiv 2,3$ & $(\bmod 4)$ & $1 / 4$ & 1.28 \\
$D \equiv 1$ & $(\bmod 8)$ & $1 / 4$ & 0.045 \\
$D \equiv 5$ & $(\bmod 8)$ & $1 / 12$ & 0.43 \\
\hline
\end{tabular}

Proof. On combining (2.14) with (3.7), (3.8), and (3.11), respectively, we find that

$$
p(D)<\mu D^{1 / 2}(A \log D+B) /(h \log \alpha) \text { for } D>64000,
$$

where $\mathrm{A}$ and $\mathrm{B}$ are given by (4.2). By a standard theorem on the class number $h$ of a quadratic field [2, p. 225], we have $2^{t} \mid h$; hence $2^{t} \leqq h$, which gives (4.1).

We now derive some corollaries; in the remainder of this section, $D$ denotes a squarefree integer $>1$.

COROLlary 1. For any fixed $\varepsilon>0$, and all sufficiently large $D$ we have

$$
p(D)<\left(A_{1}+\varepsilon\right) D^{1 / 2} \log D,
$$

where $A_{1}=1 /(4 \log \alpha)<0.52$. In particular, we have

$$
p(D)<0.52 D^{1 / 2} \log D \text { for } D>D_{1},
$$

where $D_{1}$ is a computable constant.

Proof. We have $\mu A \leqq 1 / 4$ by (4.2) and (2.5), and $2^{t} \geqq 1$. The corollary follows at once.

Corollary 2. We have

$$
p(D)<\mu 2^{-t} C(D) D^{1 / 2} \log D \text { for } \quad D>1.27 \times 10^{6},
$$

where

$$
\begin{aligned}
& C(D)=0.71 \text { for } D \equiv 2 \text { or } 3(\bmod 4), \\
& C(D)=0.53 \text { for } D \equiv 1(\bmod 8), \\
& C(D)=0.24 \text { for } D \equiv 5(\bmod 8),
\end{aligned}
$$

and we have (1.4) for $D>7$.

Proof. W obtain (4.3) from (4.1) by routine computation. This gives (1.4) for $D>1.27 \times 10^{6}$ since $\mu C(D) \leqq 0.72$ by (2.5). For $7<$ $D \leqq 1.27 \times 10^{6}$, (1.4) can be verified by use of Table 1 in [1].

COROLlary 3. The estimate (1.6) holds. 
Proof. Immediate by (4.1) or (4.3) and the definition of $t$. We remark that it can be verified, in the same way, that

$$
p(D)<0.3 D^{1 / 2} \log D \text { for } 800<D \leqq 1.27 \times 10^{6} ;
$$

this result is better than the bounds given by Corollary 1 .

We conclude this section by discussing the consequences of using (2.16) instead of (2.14) in deriving Theorem 1. We use $A_{2}$ to $A_{5}$ to denote positive constants and $E(D)$ to denote the right member of (4.1). It is clear that the use of (2.16) replaces $E(D)$ by $E(D)-$ $A_{2} \log q_{0}>E(D)-A_{3} \log D$ since $q_{0}=[\sqrt{D}]$. Now $E(D)>A_{4} D^{1 / 2} \log D 2^{-\nu}$ where $\nu$ is the number of prime factors of $D$. By a standard inequality [5, p. 262], we have $\nu<A_{5} \log D / \log \log D$. Hence $E(D)>$ $D^{1 / 2-\varepsilon}$ for any $\varepsilon>0$ and sufficiently large $D$. Thus the use of (2.16) produces only a negligible improvement in Theorem 1 for large $D$.

5. A bound for $p(D)$ when $D$ contains a square factor. We shall employ the preceding sections and elementary congruence arguments to prove the upper bound (1.5) for $p(D)$, which holds for all nonsquare integers $D>0$. Let $D$ be such an integer and set

$$
D=D_{0} s^{2},
$$

where $D_{0}$ is a fixed squarefree integer $>1$. Let $\left(a_{s}, b_{s}\right)$ be the least positive solution of $x^{2}-D y^{2}=x^{2}-D_{0} s^{2} y^{2}= \pm 1$. Put

$$
\eta_{s}=a_{s}+b_{s} \sqrt{D}=a_{s}+s b_{s} \sqrt{D_{0}},
$$

and for convenience write

$$
\eta=\eta_{1}=a+b \sqrt{D_{0}} .
$$

Now (2.7), with $\eta$ replaced by $\eta_{s}$, holds for all nonsquare $D>0$. Hence we have

$$
p(D)<\log \eta_{s} / \log \alpha .
$$

Since $\eta_{s}>1$, it follows from the theory of Pell's equation that, for fixed $D_{0}$, there is a function $e(s)>0$ such that

$$
\eta^{e(s)}=\eta_{s} \cdot
$$

Moreover, $e(s)$ is the minimum positive $k$ such that $\eta^{k}$ is congruent to a rational integer $(\bmod s)$. Hence, by $(5.4)$ and $(2.10)$, we have

$$
p(D)<\mu e(s) \log \varepsilon_{0} / \log \alpha .
$$

In $\S \S 2-4$, we showed that

$$
p\left(D_{0}\right)<\mu \log \varepsilon_{0} / \log \alpha<0.72 D_{0}^{1 / 2} \log D_{0}
$$


for $D_{0}>7$. By calculation, we find that

$$
\mu \log \varepsilon_{0} / \log \alpha<1.88 D_{0}^{1 / 2} \log D_{0}
$$

for all $D_{0} \leqq 7$. It follows from (5.1) and (5.6) that

$$
p(D)<1.88 s^{-1} e(s) D^{1 / 2} \log \left(D / s^{2}\right)
$$

for all nonsquare $D>1$.

Thus, to prove (1.5), we only need to prove $e(s) \leqq 2 s$, where $\eta$ is a unit of $Z\left[\sqrt{D_{0}}\right]$. Actually, we prove that

$$
e(s) \leqq s \quad \text { if } \quad N \eta=1
$$

and

$$
e(s) \leqq 2 s \quad \text { if } \quad N \eta=-1 .
$$

We first show that (5.8) implies (5.9). Suppose that $N \eta=-1$. Then $N \eta^{2}=1$; so, by (5.5) and (5.8), there is an integer

$$
k \leqq s \ni\left(\eta^{2}\right)^{k}=\eta^{2 k}=\eta_{s} .
$$

Hence $e(s) \leqq 2 s$, which is $(5.9)$.

Now assume that $N \eta=1$; we prove (5.8) by induction, as follows. First suppose that $s=s_{1} s_{2}$ with $\left(s_{1}, s_{2}\right)=1$. Then, by the remark after (5.5), it follows that $e(s) \leqq e\left(s_{1}\right) e\left(s_{2}\right)$. Next, suppose that $\beta \in Z\left[\sqrt{D_{0}}\right]$ and $\beta \equiv g\left(\bmod p^{i}\right)$ for a fixed $i>0$, where $p$ is prime and $g$ is an integer. Then we have $\beta^{p} \equiv g^{p}\left(\bmod p^{i+1}\right)$. Hence it only remains to prove (5.8) when $s$ is a prime $p$. We use the fact that $\eta^{-1} \in Z\left[\sqrt{\bar{D}_{0}}\right]$.

Case 1. $p=2$. Then $\eta^{2} \equiv a^{2}+D_{0} b^{2}(\bmod 2)$ by $(5.3)$.

Case 2. $\quad p>2$. We have $a^{p} \equiv a$ and $D_{0}^{(p-1) / 2} \equiv\left(D_{0} \mid p\right)$, where $\left(D_{0} \mid p\right)$ is the Legendre symbol (all congruences modulo $p$ ). Hence $\eta^{p} \equiv a+\left(D_{0} \mid p\right) b \sqrt{D_{0}}$; thus $\left(D_{0} \mid p\right)=0$ implies $\eta^{p} \equiv a$ and $e(p) \leqq p$. Next we have $\eta^{p} \equiv \eta$ or $\eta^{p} \equiv \eta^{-1}$ according as $\left(D_{0} \mid p\right)=1$ or -1 . Hence $\eta^{2 j} \equiv 1$, where $2 j=p-\left(D_{0} \mid p\right)$. Set $\eta^{j}=h+k \sqrt{D_{0}}$. Then we have $h^{2}+D_{0} k^{2} \equiv 1$. But $h^{2}-D_{0} k^{2}=N \eta^{j}=1$; hence $p \mid k$ and $\eta^{j} \equiv h$. Thus we have $e(p) \leqq p,(p-1) / 2,(p+1) / 2$ for $\left(D_{0} \mid p\right)=$ $0,1,-1$, respectively. This completes the proof of (5.8).

Actually, Mathews [13, p. 94] gives a formula which yields an explicit multiple of $e(s)$ which is $\leqq s$; a proof is given for the case $s=p$ only, and we have used his argument.

6. A conjectural lower bound for $p(D)$ when $D$ is squarefree. By (1.5), we have $p(D)=0\left(D^{1 / 2} \log D\right)$ for nonsquare $D$. It is natural 
to ask for results in the opposite direction, namely, to ask for functions $g$ such that

$$
p(D)>A g(D)
$$

for an infinity of $D$, where $A$ is a positive constant.

The tables in [1] suggest we can take $g(D)=D^{1 / 2}$; however, the best known result appears to be

$$
p(D)>A \log D,
$$

which is obtainable from the fact that

$$
p(D)=m \quad \text { for } \quad D=\frac{1}{4}\left(f_{m}+1\right)^{2}+f_{m-1}+1,
$$

where $m \not \equiv 0(\bmod 3)$, and $f_{m}$ is the $m$ th Fibonacci number; in this case, the period of $V \bar{D}$ contains $m-1$ l's followed by $f_{m}+1$. Now (6.3) is easily verified by means of (2.6), and (6.2) follows on applying the inequality $f_{n}<\alpha^{n}$ for $n \geqq 0$.

We now use an estimate due to Perron $[15, p .72]$ and a theorem of Siegel on $L(1, \chi)$ to prove

THEOREM 2. Suppose there exists an infinite sequence $S$ of squarefree numbers $D$ such that $h(D)=o\left(D^{\varepsilon / 2}\right)$ for $D$ in $S$ and all $\varepsilon>0$. Then (6.1) holds with $g(D)=D^{1 / 2-\varepsilon}$ for any $\varepsilon>0$.

REMARK. There is abundant numerical support for the truth of above hypothesis, in fact for the stronger conjecture that $h(D)=1$ infinitely often [10].

Proof. We use the following cruder form of Perron's estimate. Let $D$ be a nonsquare $>1$, and let $\left(x_{1}, y_{1}\right)$ be the least positive solution of $x^{2}-D y^{2}=1$. Then we have $x_{1}<(\sqrt{A D})^{2 p(D)}=(A D)^{p(D)}$, where $A$ is a constant. Suppose now that $D$ is squarefree. Then $\varepsilon_{0} \leqq x_{1}+y_{1} \sqrt{D}<2 x_{1}$, where $\varepsilon_{0}$ is the fundamental unit of $Q(\sqrt{D})$. Hence there is a constant $B$ such that

$$
p(D)>B \log \varepsilon_{0} / \log D=B h \log \varepsilon_{0} / h \log D .
$$

Now fix $\varepsilon>0$; by Siegel's theorem on the size of $L(1, \chi)$ and (2.12), there exists $D_{1}(\varepsilon)$ such that, for $D>D_{1}(\varepsilon)$, we have

$$
h \log \varepsilon_{0}>D^{(2-\varepsilon) / 4}
$$

(see [3, p. 130]). Hence

$$
p(D)>\frac{B D^{(2-\varepsilon) / 4}}{h \log D}
$$


for $D>D_{1}(\varepsilon)$. Theorem 2 follows by taking $D$ so large that $\log D<D^{\circ / 4}$.

\section{REFERENCES}

1. B. D. Beach and H. C. Williams, Some Computer Results on Periodic Continued Fractions, Congressus Numerantium III (1971), Proceedings of the Second Louisiana Conference on Combinatorics, Graph Theory, and Computing, pp. 133-146. MR48, \# 245.

2. H. Cohn, A Second Course in Number Theory, New York, John Wiley, 1962.

3. H. Davenport, Multiplicative Number Theory, Chicago, Markham, 1967.

4. L. E. Dickson, Introduction to the Theory of Numbers, New York, Dover, 1957.

5. G. H. Hardy and E. M. Wright, An Introduction to the Theory of Numbers, fourth edition reprinted with corrections, Oxford, 1965.

6. D. Hickerson, Length of period of simple continued fraction expansion of $\sqrt{d}$, Pacific J. Math., 46 (1973), 429-432.

7. K. Hirst, The length of periodic continued fractions, Monatsh. Math., 76 (1972), 428-435.

8. L. K. Hua, On the least solution of Pell's equation, Bull. Amer. Math. Soc., 48 (1942), 731-735.

9. P. T. Joshi, The Size of $L(1, \chi)$ for Real Nonprincipal Characters $\chi$ with Prime Modulus, J. Number Theory, 2 (1970), 58-73.

10. K. Kloss, Some number-theoretic calculations J. Res. Nat. Bur. Standards 69B (1965), 335-336.

11. W. J. Leveque, Topics in Number Theory, Vol. 1, Reading, Mass., Addison-Wesley, 1956.

12. J. E. Littlewood, On the class-number of the corpus $P(\sqrt{k})$, Proc. London Math. Soc., (2) 27 (1928), 358-372.

13. G. B. Mathews, Theory of Numbers, New York, Chelsea, n.d.

14. A. Pen and B. Skubenko, An upper bound for the period of a quadratic irrationality (Russian), Mat. Zametki, 5 (1969), 413-418.

15. O. Perron, Abschätzung der Lösung der Pellschen Gleichung, J. reine angew. Math., 144 (1914), 71-73.

16. R. G. Stanton and R. C. Mullin, Fundamentals of Calculus, Toronto, York Printing (1967).

17. R. G. Stanton and H. C. Williams, An Application of Combinatorics in Number Theory, to appear, Ars Combinatoria I (1976).

Received June 10, 1975 and in revised form July 19, 1976.

UNIVERSiTy OF MANITOBA 


\section{PACIFIC JOURNAL OF MATHEMATICS}

\section{EDITORS}

RICHARD ARENS (Managing Editor)

University of California

Los Angeles, California 90024

\section{J. DugunduI}

Department of Mathematics University of Southern California Los Angeles, California 90007

D. Gilbarg and J. Milgram

Stanford University

Stanford, California 94305

\author{
R. A. Beaumont \\ University of Washington \\ Seattle, Washington 98105
}

\section{ASSOCIATE EDITORS}
B. H. NeUmanN
F. WOLF
K. YosHIDA

\section{SUPPORTING INSTITUTIONS}

\author{
UNIVERSITY OF BRITISH COLUMBIA \\ CALIFORNIA INSTITUTE OF TECHNOLOGY \\ UNIVERSITY OF CALIFORNIA \\ MONTANA STATE UNIVERSITY \\ UNIVERSITY OF NEVADA \\ NEW MEXICO STATE UNIVERSITY \\ OREGON STATE UNIVERSITY \\ UNIVERSITY OF OREGON \\ OSAKA UNIVERSITY
}

\author{
UNIVERSITY OF SOUTHERN CALIFORNIA \\ STANFORD UNIVERSITY \\ UNIVERSITY OF HAWAII \\ UNIVERSITY OF TOKYO \\ UNIVERSITY OF UTAH \\ WASHINGTON STATE UNIVERSITY \\ UNIVERSITY OF WASHINGTON \\ AMERICAN MATHEMATICAL SOCIETY
}

The Supporting Institutions listed above contribute to the cost of publication of this Journal, but they are not owners or publishers and have no responsibility for its content or policies.

Mathematical papers intended for publication in the Pacific Journal of Mathematics should be in typed form or offset-reproduced, (not dittoed), double spaced with large margins. Please do not use built up fractions in the text of your manuscript. You may however, use them in the displayed equations. Underline Greek letters in red, German in green, and script in blue. The first paragraph or two must be capable of being used separately as a synopsis of the entire paper. Items of the bibliography should not be cited there unless absolutely necessary, in which case they must be identified by author and Journal, rather than by item number. Manuscripts, in triplicate, may be sent to any one of the editors. Please classify according to the scheme of Math. Reviews, Index to Vol. 39. All other communications should be addressed to the managing editor, or Elaine Barth, University of California, Los Angeles, California, 90024.

The Pacific Journal of Mathematics expects the author's institution to pay page charges, and reserves the right to delay publication for nonpayment of charges in case of financial emergency.

100 reprints are provided free for each article, only if page charges have been substantially paid. Additional copies may be obtained at cost in multiples of 50 .

The Pacific Journal of Mathematics is issued monthly as of January 1966. Regular subscription rate: $\$ 72.00$ a year (6 Vols., 12 issues). Special rate: $\$ 36.00$ a year to individual members of supporting institutions.

Subscriptions, orders for back numbers, and changes of address should be sent to Pacific Journal of Mathematics, 103 Highland Boulevard, Berkeley, California, 94708.

PUBLISHED BY PACIFIC JOURNAL OF MATHEMATICS, A NON-PROFIT CORPORATION

Printed at Kokusai Bunken Insatsusha (International Academic Printing Co., Ltd.), 8-8, 3-chome, Takadanobaba, Shinjuku-ku, Tokyo 160, Japan. 


\section{Pacific Journal of Mathematics}

Patricia Andresen and Marvin David Marcus, Weyl's inequality and

quadratic forms on the Grassmannian .......................

George Bachman and Alan Sultan, Regular lattice measures: mappings and

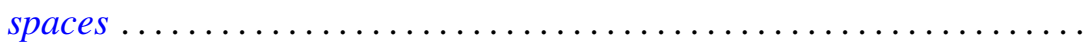

David Geoffrey Cantor, On certain algebraic integers and approximation by rational functions with integral coefficients ...................

James Richard Choike, On the value distribution of functions meromorphic in the unit disk with a spiral asymptotic value ..................

David Earl Dobbs, Divided rings and going-down................ 353

Mark Finkelstein and Robert James Whitley, Integrals of continuous

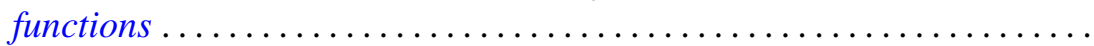

Ronald Owen Fulp and Joe Alton Marlin, Integrals of foliations on manifolds with a generalized symplectic structure ...............

Cheong Seng Hoo, Principal and induced fibrations .................

Wu-Chung Hsiang and Richard W. Sharpe, Parametrized surgery and

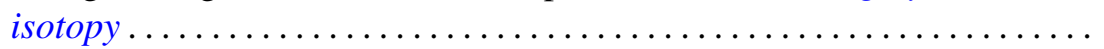

Surender Kumar Jain, Surjeet Singh and Robin Gregory Symonds, Rings whose proper cyclic modules are quasi-injective .................

Pushpa Juneja, On extreme points of the joint numerical range of commuting normal operators...

Athanassios G. Kartsatos, Nth order oscillations with middle terms of order $N-2$.

John Keith Luedeman, The generalized translational hull of a

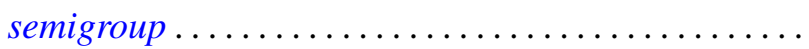

Louis Jackson Ratliff, Jr., The altitude formula and DVR's ...

Ralph Gordon Stanton, C. Sudler and Hugh C. Williams, An upper bound for the period of the simple continued fraction for $\sqrt{D}$...

David Westreich, Global analysis and periodic solutions of second order systems of nonlinear differential equations...

David Lee Armacost, Correction to: "Compactly cogenerated LCA

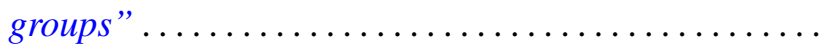

Jerry Malzan, Corrections to: "On groups with a single involution" .

David Westreich, Correction to: "Bifurcation of operator equations with unbounded linearized part" ...................... 